\title{
Polarization-independent high-index contrast grating and its fabrication tolerances
}

Ikeda, Kazuhiro; Takeuchi, Kazuma; Takayose, Kentaro; Chung, II-Sug; Mørk, Jesper; Kawaguchi, Hitoshi

\section{Published in:}

Applied Optics

Link to article, DOI:

10.1364/AO.52.001049

Publication date:

2013

Document Version

Publisher's PDF, also known as Version of record

Link back to DTU Orbit

Citation (APA):

Ikeda, K., Takeuchi, K., Takayose, K., Chung, I-S., Mørk, J., \& Kawaguchi, H. (2013). Polarization-independent high-index contrast grating and its fabrication tolerances. Applied Optics, 52(5), 1049-1053.

https://doi.org/10.1364/AO.52.001049

\section{General rights}

Copyright and moral rights for the publications made accessible in the public portal are retained by the authors and/or other copyright owners and it is a condition of accessing publications that users recognise and abide by the legal requirements associated with these rights.

- Users may download and print one copy of any publication from the public portal for the purpose of private study or research.

- You may not further distribute the material or use it for any profit-making activity or commercial gain

- You may freely distribute the URL identifying the publication in the public portal

If you believe that this document breaches copyright please contact us providing details, and we will remove access to the work immediately and investigate your claim. 


\title{
Polarization-independent high-index contrast grating and its fabrication tolerances
}

\author{
Kazuhiro Ikeda, ${ }^{1, \star}$ Kazuma Takeuchi, ${ }^{1}$ Kentaro Takayose,${ }^{1}$ II-Sug Chung, ${ }^{2}$ \\ Jesper Mørk, ${ }^{2}$ and Hitoshi Kawaguchi ${ }^{1}$ \\ ${ }^{1}$ Graduate School of Materials Science, Nara Institute of Science and Technology, 8916-5 Takayama, Ikoma, \\ Nara 630-0192, Japan \\ ${ }^{2}$ Department of Photonics Engineering, Technical University of Denmark, Kgs. Lyngby DK-2800, Denmark \\ ${ }^{*}$ Corresponding author: kazikeda@ms.naist.jp
}

Received 19 October 2012; revised 3 January 2013; accepted 5 January 2013; posted 7 January 2013 (Doc. ID 178363); published 8 February 2013

\begin{abstract}
A polarization-independent, high-index contrast grating (HCG) with a single layer of cross stripes allowing simple fabrication is proposed. Since the cross stripes structure can be suspended in air by selectively wet-etching the layer below, all the layers can be grown at once when implemented for vertical-cavity surface-emitting lasers. We optimized the structure to have a broad and high reflectivity band centered at around $1 \mu \mathrm{m}$ using a finite difference time domain method, and obtained an $80 \mathrm{~nm}$ high reflectivity band centered at $0.97 \mu \mathrm{m}$ in which the reflectivity exceeded $99.5 \%$. We also investigated the fabrication tolerances of the structure and found that, assuming careful optimizations of electron beam lithography for the precise grating width and dry-etching for the vertical sidewall, the suggested polarizationindependent HCG can be fabricated using standard technologies. @ 2013 Optical Society of America OCIS codes: $\quad 050.6624,230.4040,230.1950$.
\end{abstract}

\section{Introduction}

Recently, high-index contrast gratings (HCGs) featuring broadband reflectivity and being much thinner than conventional distributed Bragg reflectors (DBRs) have been proposed [1] and vertical-cavity surface-emitting lasers (VCSELs) exploiting the HCG have been demonstrated [2,3]. The HCG reflectors work based on guided mode resonance (GMR) under the phase-matching condition with the secondorder grating $[4,5]$. When it is used for a broadband reflector instead of a DBR in VCSELs, the effective cavity length can be significantly reduced [6], which could contribute to faster modulation. Specifically, we expect faster polarization switching in the case of polarization-bistable VCSELs [7] due to the faster cavity buildup time for lasing. To apply HCGs to polarization-bistable VCSELs, the reflectivity has to be polarization independent. Although

1559-128X/13/051049-05\$15.00/0

(C) 2013 Optical Society of America polarization-independent HCGs have been proposed [8-10], these structures are not suitable for VCSELs because of the complexity in fabrication. The twodimensional (2D) periodic islands structure $[8,9]$ requires a low-index supporting material such as $\mathrm{SiO}_{2}$ under the grating, which needs film depositions additional to the crystal growth. The cross-stacked grating [10] apparently requires more process steps to fabricate. In this paper, we propose a single layer cross-striped HCG which can be fabricated more easily using the air-bridge structure $[\underline{2}, \underline{3}]$ and can provide processing robustness for large-area HCGs, preventing grating lines from sticking to each other. We numerically investigate the structure for a polarization-independent broadband reflectivity and also the fabrication tolerances of an optimized structure.

\section{Polarization-Independent HCG}

Figure 1 shows our HCG structure, which consists of a semiconductor $2 \mathrm{D}$ grating, a low refractive index layer, and a semiconductor substrate. The 


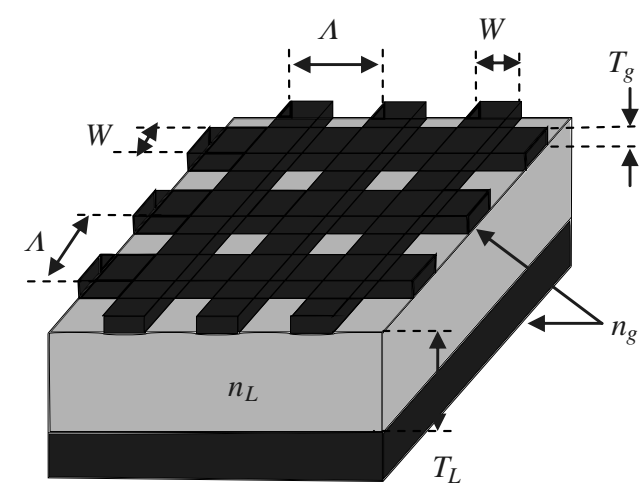

Fig. 1. Polarization-independent HCG with a single layer of cross stripes for simple fabrication.

cross-striped structure can be suspended in air by selectively wet-etching the underlayer as opposed to the 2D periodic islands structure. Therefore, all the layers for a HCG-VCSEL can be grown at once $[2,3]$. The $90^{\circ}$ rotational symmetry of the structure secures the polarization-independent reflectivity. The physical basis of the high reflectivity will be understood by the GMR for the leaky modes in the grating structure as discussed comprehensively in [5]. Since our HCG structure is intended for the upper mirror of VCSELs, the reflection from the back surface of the substrate does not need to be considered [1]. We optimized the structure to have a broad and high reflectivity band centered at around $1 \mu \mathrm{m}$ using a finite difference time domain solver, MEEP [11], where the periodic boundary condition and the perfectly matched layer are assumed in the in-plane and out-of-plane direction, respectively. Modal analysis will be another tool to investigate grating structures [12]. The high-reflectivity band can be shifted in wavelength simply by scaling the structure size. An input plane wave incident from the upper side in Fig. 1 is considered since this configuration allows simpler characterization of the fabricated HCG structure. Our calculation for the structure of Fig. 1 showed that the result considering an input wave from the bottom side is the same. We started from a cross-striped structure with the size of the onedimensional grating in [3], and found that a set of parameters, $\Lambda=556 \mathrm{~nm}, W=178 \mathrm{~nm}, T_{g}=270 \mathrm{~nm}$, $T_{L}=630 \mathrm{~nm}, n_{g}=3.5$, and $n_{L}=1$ provides an $80 \mathrm{~nm}$ high reflectivity band centered at $0.97 \mu \mathrm{m}$, in which the reflectivity exceeds $99.5 \%$, as shown in Fig. 2(a). The small ripple seen in the inset of Fig. 2(a) is an artifact of the calculation, having an amplitude that changes with the resolution setting. The bandwidth similar to DBRs and the high reflectivity are sufficient for VCSELs [2,3]. We note that cavity modes in VCSELs have oblique plane wave components and the reflectivity of HCGs is known to be sensitive to the incident angle [13]. Therefore, the reflectivity for the cavity modes in VCSELs will be lower than that presented here using a plane wave. However, our HCG structure will be still polarization independent since the cavity modes

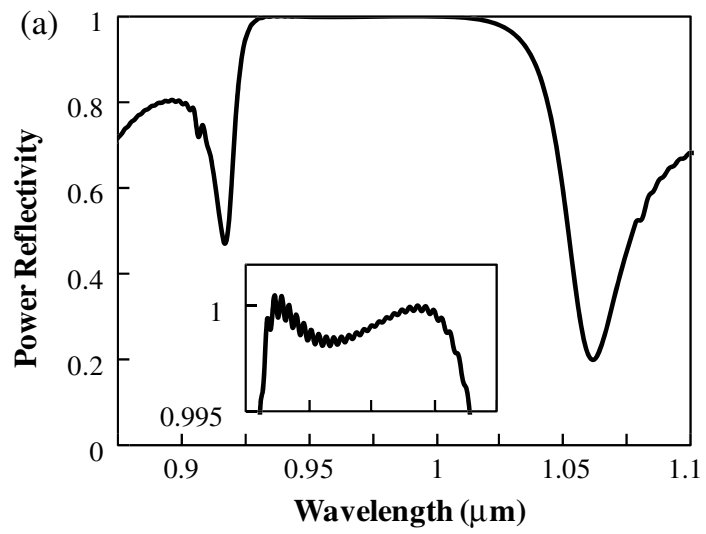

(b)

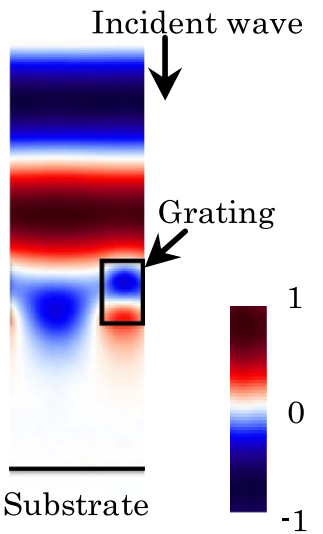

Fig. 2. (Color online) (a) Calculated power reflectivity spectrum of the optimized HCG $\left(\Lambda=556 \mathrm{~nm}, W=178 \mathrm{~nm}, T_{g}=270 \mathrm{~nm}\right.$, $T_{L}=630 \mathrm{~nm}, n_{g}=3.5, n_{L}=1$ ). (b) The electric field distribution (linear polarization perpendicular to the paper) in the unit cell for a wavelength within the high-reflectivity band.

have the cylindrical symmetry. The electric field distribution in the unit cell for a wavelength with the highest reflectivity $(\lambda=0.99 \mu \mathrm{m})$ is also presented in Fig. 2(b). We observe the field decaying below the grating.

\section{Fabrication Tolerances}

When the structure of Fig. 1 is fabricated, $W$ is the most critical, being particularly sensitive to fabrication error due to the small size. In order to evaluate the fabrication tolerance, we first calculated the $W$ dependence of the reflectivity spectrum as shown in Fig. 3(a). We see that the high reflectivity band (higher than 99.5\%) splits into two, cf. the qualitative two-hump form of the spectrum seen in the inset of Fig. 2(a), when $W$ is larger than $188 \mathrm{~nm}$ and those split bands have a quite narrow bandwidth. It is thus found from Fig. 3(a) that the fabrication tolerance for $W$ will be about $\pm 10 \mathrm{~nm}$ to keep the reflectivity higher than $99.5 \%$ at a wavelength of $0.97 \mu \mathrm{m}$. Electron beam lithography has this level of pattern definition accuracy under carefully controlled conditions [14]. Optical lithography may also achieve such pattern definition accuracy [15]. We also calculated the case with nonvertical sidewalls as presented in Fig. 3(b). The definition of the 

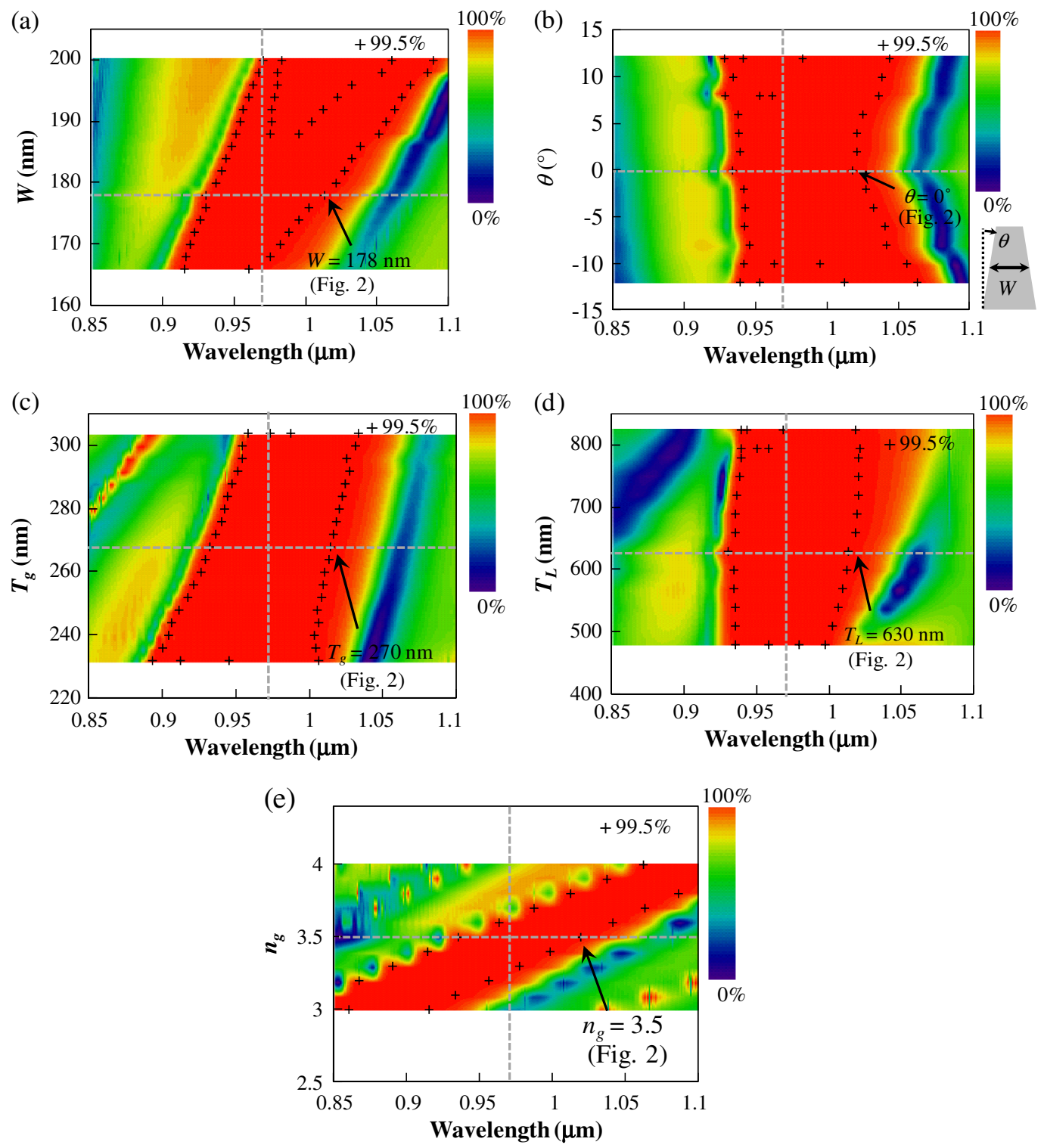

Fig. 3. (Color online) (a) $W$, (b) $\theta$, (c) $T_{g}$, (d) $T_{L}$, and (e) $n_{g}$ dependences of the reflectivity spectrum. The other parameters are the same as those in Fig. 2. The symbol "+" indicates $99.5 \%$.

tilted angle $\theta$ is described in the inset of Fig. 3(b). We observe that the high reflectivity band splits into two when $\theta$ is larger than $\pm 8^{\circ}$. It is thus found from Fig. 3(b) that the fabrication tolerance for $\theta$ will be about $\pm 6^{\circ}$. This level of vertical dry-etching is also possible under carefully controlled conditions [16]. Similar considerations for the other structural parameters resulted in $T_{g}=270 \pm 30 \mathrm{~nm}$, $T_{L}=630 \pm 130 \mathrm{~nm}$, and $n_{g}=3.5 \pm 0.1$, respectively, as shown in Figs. 3(c)-3(e). Since we assume that the grating and low index layers are all grown with molecular beam epitaxy (MBE), the above tolerances of $T_{g}$ and $T_{L}$ are not critical. It is worth noting that if the MBE growth has a $\pm 10 \mathrm{~nm}$ thickness error, different choices of $T_{g}$ and $T_{L}$ will be possible. For example, $T_{g}=250 \mathrm{~nm}$ results in a wider bandwidth of $\sim 100 \mathrm{~nm}$ as seen in Fig. 3(c), and $T_{L}=$ $510 \mathrm{~nm}$ provides a shorter effective cavity length although the bandwidth becomes slightly narrower, as seen in Fig. 3(d). Finally, the above tolerance of $n_{g}$ is not critical either since the composition of the grating material (e.g., $\mathrm{Al}_{x} \mathrm{Ga}_{1-x} \mathrm{As}$ ) can be well controlled by MBE.

It is interesting to consider the effect of an asymmetric fabrication error on the polarizationindependent reflectivity. Our structure is polarization independent because of the $90^{\circ}$ rotational symmetry. However, an asymmetric fabrication error, for example, $W_{x}=178+5 \mathrm{~nm}, W_{y}=178-5 \mathrm{~nm}$, results in a polarization-dependent reflectivity as shown in Fig. 4 . We see that the high reflectivity bands for $E_{x}$ and $\bar{E}_{y}$ linear polarizations shift in the same manner as in Fig. 3(a), but have an overlap of between 0.95 and $1 \mu \mathrm{m}$, where the polarization-independent property almost holds. When applied to VCSELs, the maximum reflectivity difference at $0.98 \mu \mathrm{m}$, i.e., 


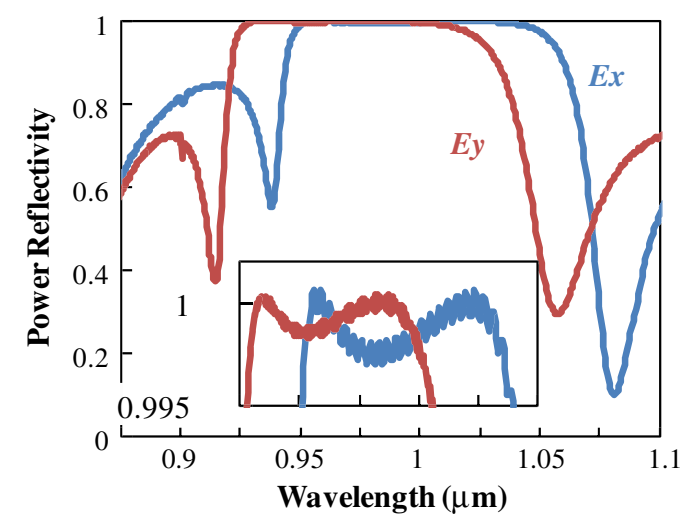

Fig. 4. (Color online) Calculated power reflectivity spectra (for $E_{x}$ and $E_{y}$ linear polarizations) of the optimized HCG with an asymmetric fabrication error, $W_{x}=183 \mathrm{~nm}, W_{y}=173 \mathrm{~nm}$.

$R_{\mathrm{HCG}}=\sim 0.997$ and $\sim 0.999$ for $E_{x}$ and $E_{y}$ polarizations, respectively, results in an $8 \%$ difference of lasing threshold gains $g_{\text {th }}$, assuming a simple relation of $g_{\text {th }}=\alpha+1 /(2 d) \cdot \ln \left(1 / \sqrt{R_{\mathrm{HCG}} \cdot R_{\mathrm{DBR}}}\right)$ with a loss inside the cavity of $\alpha=20 \mathrm{~cm}^{-1}$, a reflectivity of the opposing DBR mirror of $R_{\mathrm{DBR}}=0.999$, and a cavity length of $d=3 \mu \mathrm{m}$ [17]. Further optimization to minimize the dent in the high-reflectivity band will be possible for more robust polarization independence.

Finally, we investigate the effect of absorption in the grating material on the reflectivity. We assume that the absorption originates from carriers unintentionally ( $p$-type at $2 \times 10^{14} \mathrm{~cm}^{-3}$ ) or intentionally ( $p$-type at $2 \times 10^{16} \mathrm{~cm}^{-3}$ ) doped in MBE growth. The absorption coefficients at the doping levels above are calculated as $\alpha=0.4$ and $29.3 \mathrm{~cm}^{-1}$, respectively, using the corresponding mobilities in $\mathrm{Al}_{0.6} \mathrm{Ga}_{0.4} \mathrm{As}$ [18], which is a typical material used in air-bridge $\mathrm{HCG}-\mathrm{VCSELs}$ $[2,3]$. The power reflectivity spectra calculated using the absorption coefficients are plotted in Fig. 5. We see that the carrier density intrinsically included in MBEgrown crystals $\left(\sim 2 \times 10^{14} \mathrm{~cm}^{-3}\right)$ does not change the reflectivity spectrum, while the carrier density intentionally doped $\left(2 \times 10^{16} \mathrm{~cm}^{-3}\right)$ reduces the reflectivity.

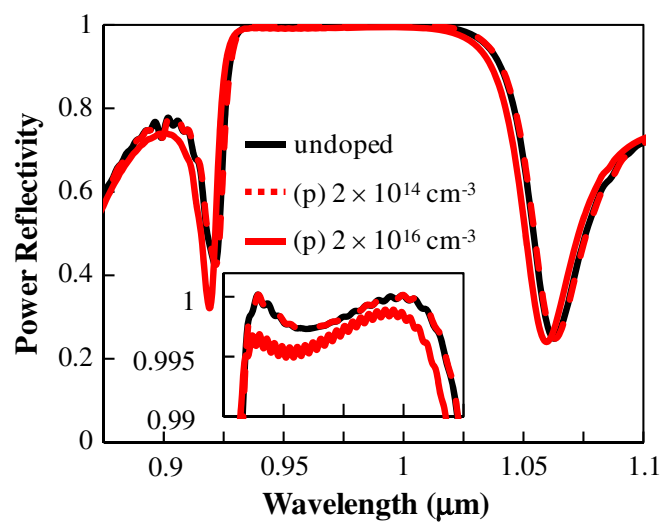

Fig. 5. (Color online) Calculated power reflectivity spectra of the optimized HCG with the absorption coefficients of 0.4 and $29.3 \mathrm{~cm}^{-1}$ at the doping levels of (p) $2 \times 10^{14} \mathrm{~cm}^{-3}$ and (p) $2 \times 10^{16} \mathrm{~cm}^{-3}$, respectively.
From the inset of Fig. 5, a carrier density higher than $2 \times 10^{16} \mathrm{~cm}^{-3}$ will result in a reflectivity lower than 99.5\% which makes an impact on the lasing threshold gain in HCG-VCSELs.

\section{Conclusions}

We have proposed a polarization-independent HCG with a single layer of cross stripes for simple fabrication. An $80 \mathrm{~nm}$ high-reflectivity band centered at a wavelength of $0.97 \mu \mathrm{m}$, in which the reflectivity exceeds $99.5 \%$, was obtained using optimized structural parameters. The bandwidth is similar to that realized by DBR mirrors and the reflectivity achieved is sufficient for VCSELs. We have also investigated the fabrication tolerances of the structure and found that, assuming careful optimizations of electron beam lithography for the $W$ tolerance of $\pm 10 \mathrm{~nm}$ and dry-etching for the $\theta$ tolerance of $\pm 6^{\circ}$, the polarization-independent HCG can be fabricated using standard technologies. In addition, it has been found that material absorption originating from carriers intrinsically included in MBE-grown crystals $\left(\sim 2 \times 10^{14} \mathrm{~cm}^{-3}\right)$ does not reduce the reflectivity, while a carrier density higher than $2 \times 10^{16} \mathrm{~cm}^{-3}$ will result in a reflectivity lower than $99.5 \%$, which makes an impact on the lasing threshold gain in HCG-VCSELs.

This work was supported in part by the "R\&D promotion scheme funding international joint research" promoted by the NICT (National Institute of Information and Communications Technology).

\section{References}

1. C. F. R. Mateus, M. C. Y. Huang, D. Yunfei, A. R. Neureuther, and C. J. Chang-Hasnain, "Ultrabroadband mirror using low-index cladded subwavelength grating," IEEE Photon. Technol. Lett. 16, 518-520 (2004).

2. M. C. Y. Huang, Y. Zhou, and C. J. Chang-Hasnain, "A surface emitting laser incorporating a high-index-contrast subwavelength grating," Nat. Photonics 1, 119-122 (2007).

3. Y. Zhou, M. C. Y. Huang, and C. J. Chang-Hasnain, "Tunable VCSEL with ultra-thin high contrast grating for high-speed tuning," Opt. Express 16, 14221-14226 (2008).

4. A. Hardy, D. F. Welch, and W. Streifer, "Analysis of secondorder gratings," IEEE J. Quantum Electron. 25, 2096-2105 (1989).

5. R. Magnusson and M. Shokooh-Saremi, "Physical basis for wideband resonant reflectors," Opt. Express 16, 3456-3462 (2008).

6. I.-S. Chung, V. Iakovlev, A. Sirbu, A. Mereuta, A. Caliman, E. Kapon, and J. Mørk, "Broadband MEMS-tunable highindex-contrast subwavelength grating long-wavelength VCSEL,” IEEE J. Quantum Electron. 46, 1245-1253 (2010).

7. H. Kawaguchi, T. Mori, Y. Sato, and Y. Yamayoshi, "Optical buffer memory using polarization bistable vertical-cavity surface-emitting lasers,” Jpn. J. Appl. Phys. 45, L894-L897 (2006).

8. J. Yamauchi, N. Goto, and H. Nakano, "Broadband mirror using a two-dimensional subwavelength grating," in Proceedings of the Society Conference of IEICE, Electronics (IEICE, 2007), p. 196 (in Japanese)

9. R. G. Mote, S. F. Yu, W. Zhou, and X. F. Li, "Design and analysis of two-dimensional high-index-contrast grating surfaceemitting lasers," Opt. Express 17, 260-265 (2009).

10. D. Zhao, H. Yang, Z. Ma, and W. Zhou, "Polarization independent broadband reflectors based on cross-stacked gratings," Opt. Express 19, 9050-9055 (2011). 
11. A. F. Oskooi, D. Roundy, M. Ibanescu, P. Bermel, J. D. Joannopoulos, and S. G. Johnson, "MEEP: a flexible freesoftware package for electromagnetic simulations by the FDTD method," Comput. Phys. Commun. 181, 687-702 (2010).

12. S. K. Chin, N. A. Nicorovici, and R. C. McPhedran, "Green's function and lattice sums for electromagnetic scattering by a square array of cylinders," Phys. Rev. E 49, 4590-4602 (1994).

13. H. Sano, J. Kashino, A. Gerke, A. Imamura, F. Koyama, and C. Chang-Hasnain, "Transverse mode control of VCSELs with high contrast sub-wavelength grating functioning as angular filter," in 2012 Conference on Lasers and Electro-Optics (IEEE, 2012), paper CW3N.5.

14. E. Kuramochi, M. Notomi, S. Mitsugi, A. Shinya, T. Tanabe, and T. Watanabe, "Ultrahigh-Q photonic crystal nanocavities realized by the local width modulation of a line defect," Appl. Phys. Lett. 88, 041112 (2006).

15. M. Settle, M. Salib, A. Michaeli, and T. F. Krauss, "Low loss silicon on insulator photonic crystal waveguides made by $193 \mathrm{~nm}$ optical lithography," Opt. Express 14, 2440-2445 (2006).

16. M. Notomi, K. Yamada, A. Shinya, J. Takahashi, C. Takahashi, and I. Yokohama, "Extremely large group-velocity dispersion of line-defect waveguides in photonic crystal slabs," Phys. Rev. Lett. 87, 253902 (2001).

17. K. Iga, F. Koyama, and S. Kinoshita, "Surface emitting semiconductor lasers," IEEE J. Quantum Electron. 24, 1845-1855 (1988).

18. M. Sotoodeh, A. H. Khalid, and A. A. Rezazadeh, "Empirical low-field mobility model for III-V compounds applicable in device simulation codes," J. Appl. Phys. 87, 2890-2900 (2000). 